\title{
Diabetes and metabolic complications among patients with HIV and AIDS
}

\author{
M I Weerakkody ${ }^{1}$, K Buddhakorala ${ }^{2}$, N P Somasundaram ${ }^{3}$ \\ Sri Lanka Journal of Diabetes, Endocrinology and Metabolism 2013; 3: 68-75
}

\begin{abstract}
HIV infection, as well as its treatment with highly active retroviral therapy (HAART), is associated with a number of metabolic abnormalities such as insulin resistance, glucose intolerance and dyslipidaemia. The aim of this descriptive cross sectional study was to describe the prevalence of diabetes, impaired fasting glucose, dyslipidaemia and obesity among patients with HIV or AIDS who were followed up at the National Sexually Transmitted Diseases (STD) campaign in the year 2010, and to assess the associated factors.

A total of 268 patients were evaluated of which $57.8 \%$ were male. Fifty six percent were on HAART therapy. Eighteen patients (6.7\%) were diabetics and forty nine (18.3\%) had impaired fasting glucose. Only about $40 \%$ of the diabetic patients were followed up regularly. About $20 \%$ of the patients had high total cholesterol levels (>240mg/dl) and only $24.4 \%$ patients had an optimal LDL cholesterol level of $<100$ $\mathrm{mg} / \mathrm{dl} .15 .3 \%$ of the patients had high serum triglyceride levels $(200-499 \mathrm{mg} / \mathrm{dl})$ and $1.9 \%$ had very high triglyceride levels $(>500 \mathrm{mg} / \mathrm{dl}$ ). Fourteen percent of the patients were overweight and $18.2 \%$ of the patients were obese according to the WHO criteria for body mass index for Asians. Being on HAART therapy was significantly associated with having high total cholesterol levels, high LDL cholesterol levels and high triglyceride cholesterol levels but not with elevated blood glucose values in the $X^{2}$ test $(p<0.05)$. The mean total cholesterol, LDL cholesterol and triglyceride levels were also significantly different among HAART users and non users. Having HIV for more than 3.1 years (more than the median) and duration of HAART therapy for more than 1.9 years (more than the median) were also significantly associated with high total cholesterol and LDL cholesterol levels $(p<0.05)$.
\end{abstract}

Metabolic abnormalities are common among HIV infected persons and some of them are associated with the duration of HIV and the use of HAART therapy.

\section{Introduction}

The introduction of the highly active antiretroviral therapy (HAART) has changed the course of HIV infection, increasing survival and improving quality of life in HIV-infected individuals. The search for new therapeutic modalities and management strategies has reduced the acute morbidity of the disease but has created a number of chronic effects. A number of metabolic abnormalities are associated with HIV management; the most common being insulin resistance, glucose intolerance, dyslipidaemia, changes in body fat distribution and osteopenia.

Insulin resistance rather than insulin deficiency is responsible for pathogenesis of diabetes in HIV infected persons. Increased levels of intrahepatic tumour necrosis factor and hepatic steatosis leads to insulin resistance and diabetes, even in patients not on HAART therapy (1). The major contributor to hyperglycemia in HIV/AIDS however, is drugs. Highly active retroviral therapy
(HAART) has contributed immensely to the remarkable clinical outcome of HIV patients, but it has also lead to an increase in metabolic dysfunction, insulin resistance, diabetes dyslipidaemia and lipodystrophy (2). A recent analysis found that diabetes is four fold more common in HIV infected men exposed to highly active anti retroviral therapy (HAART) than in HIV seronegative men (3). Diabetes may also be caused by drugs used to manage co morbidities associated with HIV such as Pentamidine used for Pneumocystis Jerovecii infection and Megesterol acetate used as an appetite stimulant $(4,5)$.

During the course of HIV infection and acquired immunodeficiency syndrome (AIDS), disturbances of lipid metabolism were observed long before the introduction of Protease Inhibitor (PI) based antiretroviral regimens, and included hypertriglyceridaemia and a decrease in total and high-density lipoprotein (HDL) cholesterol, occurring in advanced phases of HIV infection (6). Even though therapy with zidovudine, lamivudine, stavudine, or non-

${ }^{1}$ Senior Registrar in Endocrinology, ${ }^{3}$ Consultant Endocrinologist, Diabetes and Endocrine Unit, The National Hospital of Sri Lanka, ${ }^{2}$ Consultant Venereologist, National STD and AIDS Campaign, Sri Lanka. 
nucleoside-reverse transcriptase inhibitors (NNRTIs) has been associated with the occurrence of dyslipidaemia, abnormalities of plasma lipid levels appear to be most prevalent among patients receiving a PI-based regimen and ranges from $28 \%$ to $80 \%$ (6). Among non-PI drugs, Efavirenz and Stavudine lead to Triglyceride elevation, whereas Tenofovir may reduce the Triglyceride level. LDL$\mathrm{C}$ levels are increased in association with most PI- and NNRTI-based regimens (7).

Treatment of these cardiovascular risk factors is of paramount importance in the management of HIV, because studies show that the relative rate of MI hospitalization in HIV-infected persons was more than 2-fold greater than that of controls (7). Management of metabolic complications poses a number of challenges in co existent HIV infection. Poly pharmacy, co existing renal and hepatic disease, induction of cytochrome induction pathways with certain antiretroviral drugs causing toxicity of drugs such as statins, the necessity for strict adherence to universal precautions with insulin therapy and the reluctance of caregivers for invasive procedures like administering insulin are some of them. These patients would also be reluctant to seek medical advice or comply for long-term follow-up for these metabolic problems due to the social stigma that is still associated with HIV infection in this part of the world.

Sri Lanka has about 1600 reported HIV infected persons at present with an estimated 4200 of both reported and unreported cases (8). They are followed up in several clinics around the country, the clinic at the National Sexually Transmitted Diseases (STD) Campaign has the largest population of patients, it has followed up a total of 820 patients since its inception in 1997. A total of 369 patients had attended services in the clinic in 2010. Prevalence of diabetes and metabolic abnormalities among patients with HIV/AIDS in Sri Lanka has not been assessed previously.

The aim of this study was to describe the prevalence of diabetes, impaired fasting glucose and other metabolic complications such as dyslipidaemia and obesity among the HIV infected patients being followed up at the National STD Campaign. It was also aimed at assessing any factors associated with the above metabolic parameters and assessing any correlation between antiretroviral therapy and metabolic parameters.

\section{Method}

This was a descriptive cross sectional study carried out at the National STD Campaign clinic in Colombo. The HIV patients who had attended services at the above clinic during the calendar year 2010 were recruited to the study. This included newly diagnosed patients as well as patients who were diagnosed prior to that but had attended the clinic during 2010. All HIV-infected patients aged 18 and older were included in the sample regardless of being on HAART or not but patients who had not had at least two clinic visits in 2010 were excluded. Ethical approval was obtained from the Ethical Review Committee, Faculty of Medicine, University of Colombo.

As the patients in the clinic routinely undergo biochemical screening at least 3 to 6 monthly, the data on fasting blood glucose and serum lipids were obtained from these records. The anthropometric data (height, weight), which are routinely recorded by the trained nursing officer at the clinic was also obtained from patient records. Data on HAART was taken from medical records and patients were considered to be HAART positive if they were on drugs for at least two months.

Diabetes and impaired fasting glucose were defined according to the ADA 2012 criteria (9), lipids were categorized according to NCEP-ATP3 (10) criteria, and obesity was described according to the WHO classification of obesity for Asians (11). Descriptive statistics were used according to the distribution of the variable: for variables with a normal distribution, occurrence measures, mean and standard deviation values were shown; for non-parametric variables, quartile ranges and median values were shown. Independent sample t test was used to compare means and the associations between metabolic parameters and patient and disease related factors were assessed using the Pearson's chi-square test. Multivariate linear regression analysis was used to assess the contribution of the different patient, disease and treatment related factors to the metabolic parameters. The $\mathrm{P}$ value was considered to be significant at $<0.05$.

\section{Results}

This study included 268 patients with HIV or AIDS, $57.8 \%$ were males (no $=155)$. The median age was 41 years and the ages ranged from 20 to 73 years. One hundred and fifty one patients were on antiretroviral therapy (56.3\%) and the commonest combination of drugs was Zidovudine, Lamivudine and Efavirenz (2 nucleoside reverse transcriptase inhibitors and 1 non nucleoside reverse transcriptase inhibitors), $52 \%$ of the patients had been on this combination for more than 2 months. Only 7.2\% were on protease inhibitor based HAART regimes.

This population had 18 diabetic patients (6.7\%) and 08 (3\%) of them were known diabetics at the diagnosis of HIV. Forty nine patients (18.3\%) had impaired fasting glucose by the ADA definition. Only 7 (38.9\%) of the diabetic patients were on regular follow-up regarding diabetes; however $78 \%$ were taking either oral hypoglycaemic drugs or insulin. Only 6 (33.3\%) had target organ screening done and half of those patients had evidence of microvascular disease. 
Table 1. Basic, disease and treatment characteristics of patients

Total no of patients

Gender

male

$155(57.8 \%)$

female

113(42.2)

Median age in years (25-75 range)

41(33-46)

Smoking status

Current smoker

$54(20.1 \%)$

Past smoker

$10(3.7 \%)$

Never smoked

$204(76.1 \%)$

Substance abuse

12 (4.5\%)

Mean CD4 count at HIV diagnosis

$390(242.7)$

$<200$

$59(24.8 \%)$

200-500

$104(43.7 \%)$

$>500$

75(31.5\%)

Median Duration of HIV in years (25-75 range)

$3.12(1.5-5.58)$

Median Duration of clinic follow-up years (25-75 range)

$2.67(1.1-5.22)$

On anti retroviral therapy

$151(56.3 \%)$

Median Duration of anti retroviral therapy - years (25-75 range)

$1.91(0.83-4.67)$

Type of anti retroviral therapy

Nucleoside reverse transcript inhibitors

145(96\%)

Non nucleoside reverse transcript inhibitors

$143(94.7 \%)$

Nucleotide reverse transcript inhibitors

$22(14.6 \%)$

Protease inhibitors

$11(7.2 \%)$

Diabetics

18(6.7\%)

Known diabetics at HIV diagnosis

$08(3.0 \%)$

Diabetes diagnosed during follow up

$10(3.7 \%)$

Impaired fasting glucose

$18(3 \%)$

On Statin therapy for $>3$ months

$19(07 \%)$

The metabolic characteristics of the population studied is described in Table 3.

Forty nine (23.6\%) of the non diabetic population had impaired fasting glucose and $78 \%$ of the diabetic patients (no=14) had fasting blood glucose above 110 $\mathrm{mg} / \mathrm{dl}$ despite being on treatment.

About $20 \%$ of the patients had high total cholesterol levels (>240mg/dl) and only $24.4 \%$ patients had an optimal LDL cholesterol level of $<100 \mathrm{mg} / \mathrm{dl}$. $15.3 \%$ of the patients had high serum triglyceride levels (200-499mg/dl) and 1.9\% had very high triglyceride levels above $500 \mathrm{mg} / \mathrm{dl}$. Only $12.2 \%(n o=27)$ of the patients had low HDL cholesterol levels of less than $40 \mathrm{mg} / \mathrm{dl}$.

One hundred and eight patients (44.6\%) had normal body mass index (BMI) according to the WHO classification of body mass index for Asians (11). However $14 \%$ of the patients were overweight and 18.2\% of the patients were obese according to the same criteria. 

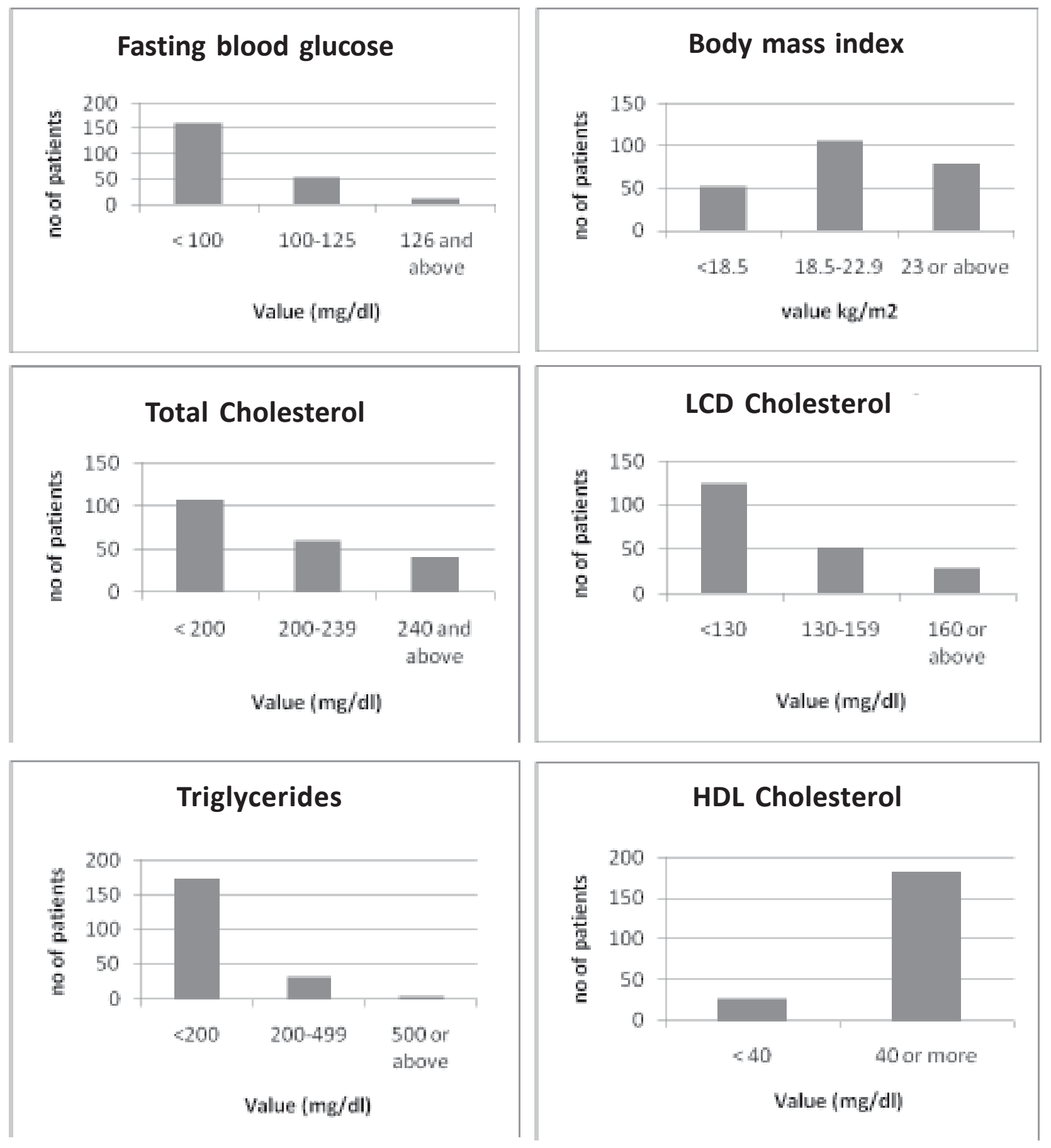

Figure 1. Metabolic characteristics of patients.

The associations between various metabolic parameters and patient and disease characteristics such as age, gender, smoking habits, body mass index, lowest CD4 count at diagnosis, duration of HIV, whether on HAART therapy and duration of HAART therapy was also assessed.

Being on HAART therapy was significantly associated with having high total Cholesterol levels, high LDL cholesterol levels and high triglyceride cholesterol levels in the $\mathrm{X}^{2}$ test $(\mathrm{p}<0.05)$. The mean total cholesterol,
LDL cholesterol and triglyceride levels were also significantly different among HAART users and non users (Table 3). Having HIV for more than 3.1 years (more than the median) and duration of HAART therapy for more than 1.9 years (more than the median) were also significantly associated with high total cholesterol and LDL cholesterol levels $(p<0.05)$. However, when the multiple regression model was used, none of the above characteristics were associated with high total cholesterol, LDL cholesterol or triglyceride levels. 
Table 2. Metabolic parameters among HAART users and non-users

\begin{tabular}{llcl}
\hline & $\begin{array}{c}\text { On HAART therapy } \\
\text { Mean (SD) }\end{array}$ & $\begin{array}{c}\text { Not on HAART therapy } \\
\text { Mean (SD) }\end{array}$ & $P$ \\
\hline Fasting blood glucose (mg/dl) & $97.47(29.53)$ & $90.88(21.11)$ & 0.24 \\
Total cholesterol (mg/dl) & $212.94(44.98)$ & $187.64(33)$ & 0.006 \\
Triglycerides (mg/dl) & $172.56(131.25)$ & $118.24(52.21)$ & 0.000 \\
LDL cholesterol (mg/dl) & $128.86(38.32)$ & $117.65(28.46)$ & 0.008 \\
HDL cholesterol (mg/dl) & $51.09(10.2)$ & $46.25(8.75)$ & 0.831 \\
Body mass index $\left(\mathrm{kg} / \mathrm{m}^{2}\right)$ & $21.72(3.96)$ & $21.09(38.4)$ & 0.28 \\
\hline
\end{tabular}

Statistics: independent sample t test, $\mathrm{p}<0.05$ significant

\section{Discussion}

This descriptive study, the first to be done on HIV patients in Sri Lanka, describes a prevalence of 6.7\% for diabetes and $18.3 \%$ for impaired fasting glucose in this population. There was a baseline prevalence of diabetes in $3 \%$ of this study population. In the Sri Lanka Diabetes Cardiovascular Study, a population based study involving 7 provinces of Sri Lanka, the prevalence of diabetes and impaired fasting blood glucose were $10.3 \%$ and $4.4 \%$ respectively (12). Our population had lower prevalence of diabetes but the prevalence of impaired fasting glucose was about 3 times higher (12). However the mean age of that population was 46.1(standard deviation 15), while our population had a lower mean age of 40.3 years (standard deviation 9.3). Previous studies assessing the prevalence and the incidence of diabetes and impaired glucose tolerance have shown a wide range of values. DAD study, one of the largest, multicentre cohort studies designed to assess the association between HAART therapy and cardiovascular disease, had a baseline prevalence of diabetes in $2.85 \%$ of the population. The new onset diabetes was 2.2\% (5.72 at 1000 person year follow up) (13). Other smaller studies with heterogeneous study designs, which included patients with and without HAART therapy, reported a prevalence of diabetes ranging from $4.5 \%$ to $7 \%$, and impaired fasting glucose ranging from $17 \%$ to $35 \%(14,15,16)$. Although most studies show that the prevalence of diabetes and impaired glucose tolerance is higher in HIV infected patients than in the normal population, a few studies have shown that there is no difference (17). In Nutrition for Healthy Living Study, a large multicentre cohort study in the US, a non diabetic subgroup was analyzed to assess the prevalence of insulin resistance by using a number of insulin resistance indices and serum insulin levels and was compared with the national NHANES111 database. The prevalence of insulin resistance varied from $17 \%-51 \%$ depending on the indices used, but it was not different to the control population in the NHANES111 (17). The wide variation of glucose parameters in different studies could be due to the different study designs, inclusion of both HAART positive and negative populations, different types of HAART drugs used (sp Protease Inhibitors), the characteristics of the control group and patient characteristics such as the presence of lipodystrophy. Some studies have used basic biochemical parameters such as fasting blood glucose and the oral glucose tolerance test whereas other studies have used measures of insulin resistance such as the HOMA index and QUICKI score. While some control groups consisted of healthier people such as blood donors other controls have been taken from national databases.

Studies have also demonstrated that the prevalence of diabetes and impaired fasting glucose is higher among HAART users when compared to HAART non users ( 3 , $13,18)$. The most commonly studied drug to be associated with HAART therapy is Protease Inhibitors. Several large scale studies among HIV patients describe a prevalence of impaired glucose tolerance ranging from 8-46\% among Protease Inhibitor users (19) out of which Ritonovir is found to be the most commonly associated agent $(3,13)$. Others studies have demonstrated that the use of Nucleoside Reverse Transcriptase Inhibitors (especially Stavudine) is associated with a higher incidence of glucose abnormalities $(20,21)$. Other factors that were associated with diabetes mellitus and impaired glucose tolerance were patient characteristics such as age, gender, body mass index and family history of diabetes mellitus and disease related factors such co infection with hepatitis $\mathrm{C}$, duration exposure to HAART therapy and CD4 count of $<300$ cells/ $\mathrm{m}^{3}$ at the index visit $(3,13,20)$. In our study there was no statistical difference in the mean fasting blood glucose levels among the HAART users and non users. Factors such as the CD4 count at diagnosis, the duration of HIV infection, and the duration of antiretroviral therapy were 
also not associated with elevated blood glucose levels either. One explanation for not finding an association between HAART use and elevated blood glucose levels in our study could be that only $7.2 \%$ of our patients were on Protease Inhibitors and only $11 \%$ were on Stavudine, the most commonly cited drugs to be associated with blood glucose abnormalities. However even in our patient population, 35.5\% of the Stavudine treated patients had impaired glucose tolerance (vs. $18 \%$ of the study population) and $27.3 \%$ of the Protease Inhibitor group had fasting blood blood glucose $>126 \mathrm{mg} / \mathrm{dl}$ (vs. $4.1 \%$ of the study population).

It is important to note that only $38 \%$ of the diabetic patients had regular follow-up regarding diabetes and $22.2 \%$ were not taking any form of therapy for the illness. Target organ screening was done only on 6 (33.3\%) of the diabetic population. Factors such as experience of drug interactions, polypharmacy, social stigma and unawareness regarding the long-term complications of diabetes may be responsible for the poor compliance to therapy. These issues need further evaluation and planning out of possible solutions.

The classic dyslipidaemia associated with HIV infection, with high total cholesterol, LDL and triglyceride levels and low HDL cholesterol is also shows different prevalence in different studies. In a French study of 925 patients, 70 patients (8\%) experienced high triglyceride levels after 25 months of follow up, and a prospective population study from Canada quotes a cumulative incidence of dyslipidaemia of $9 \%$ within 31 months of commencing HAART therapy (19). Studies in other parts of the world show higher prevalence and incidence of dyslipidaemia. In a comparison study in Brazil $41.4 \%$ and $20.5 \%$ of the patients on HAART showed high triglyceride and total cholesterol levels respectively. Compared to HIV patients not on HAART this was statistically significant (22). The same study shows that all HAART regimes can alter the lipid parameters although Ritonovir based therapy had the highest risk (22). Another study in Thai patients showed that patients on Protease inhibitors had higher Total cholesterol, Triglyceride and low HDL levels (42.6\%, $75.4 \%, 31.2 \%$ respectively) when compared to patient not on treatment (4.8\%, $8.2 \%, 42.95 \%$ respectively). Patients on other HAART drugs had in-between values of $26.6 \%$, $48.3 \%$ and $20 \%$ respectively (23). Although less well studied, other HAART drugs such as nucleoside reverse transcriptase inhibitors (NRTIs), Nonnucleoside reverse transcriptase inhibitors (NNRTIs), mainly Efavirenz might also be associated with metabolic disorders, including dyslipidaemia (24). In our study $15.3 \%$ of the patients had high ( $>240 \mathrm{mg} / \mathrm{dl}$ ) total cholesterol, 13.4\% high triglyceride ( $>200 \mathrm{mg} / \mathrm{dl}$ ) and only $10.1 \%$ had low HDL cholesterol (40 mg/dl). The somewhat lower values in our study population may be due to the low use of protease inhibitors in our patients (7.2\%). However among our patients who were on Protease Inhibitors, 63\% had total cholesterol
$>240 \mathrm{mg} / \mathrm{dl}$ and $45 \%$ had triglycerides > $200 \mathrm{mg} / \mathrm{dl}$ compared to $22 \%$ and $14.2 \%$ of patients who were on other HAART drugs. The onset of dyslipidaemia is said to be between 3 to 12 months in Protease Inhibitor based regimes (6) but the onset of dyslipidaemia with other drugs is not well documented. Therefore, as the median duration of HAART treatment (with mainly non Protease Inhibitor based therapy) is only 1.9 years in our study, that may be one reason to get lower lipid values than in certain studies. Most other studies in literature assessing the prevalence of hyperlipedaemia has either excluded the patients on lipid lowering therapy or has not mentioned whether they have done so. $7 \%$ of our patients were on treatment for hyperlipidaemia, which may have contributed to the lower values of lipid abnormalities in our study compared to some other Asian and South American studies. However, the mean total cholesterol, triglyceride and LDL levels were significantly higher in patients with HAART therapy in our study, when compared to patients not on HAART therapy. The duration of HIV infection for more than 3.1 years and being on HAART therapy for more than 1.9 years were significantly associated with having abnormal lipid levels as well.

In this patient population, $14 \%$ were overweight and $18.2 \%$ were obese according to the WHO classification of obesity for Asians. Overweight and obese patients in patients younger than 40 years were $10.3 \%, 14.3 \%$ and in patients 40 years of age or older were $14.8 \%$, and $18.3 \%$ respectively. A descriptive study in 7 provinces of Sri Lanka revealed that $25.2 \%$ of people were in overweight and $9.2 \%$ were in the obese categories using the WHO criteria for Asians (25), which are higher figures than in our study. Age and gender related figures were also higher in that study. In the D.A.D sub study assessing metabolic syndrome as a cardiovascular risk factor, only $4.4 \%$ of the sample had BMI $>30 \mathrm{~kg} / \mathrm{m}^{2}$ (26). Studies elsewhere in the world have shown prevalence of obesity ranging from $4 \%$ to $35 \%(27,28)$. Studies have also shown that waist circumferences were lower in HIV-infected HAART recipients compared with the uninfected population despite having similar prevalence rates for metabolic syndrome. In one study, although $50 \%$ of HIV-infected HAART recipients met non-anthropometric criteria for metabolic syndrome, this reduced to $17 \%$ when waist-based anthropometric cutoffs were applied (29). Therefore HIV infected patients, although having other metabolic risk factors may not be an obese population. The possible explanations for these findings could be the presence of co-existing chronic infections such as tuberculosis, malabsorption syndromes associated with the disease as well as HAART, and although much rarer now, the HIV wasting syndrome resulting in lower body mass indices. The differences in overweight and obesity prevalence among studies may also be influenced by the differences in ethnicity, gender, socio-economic status and the nutritional status of the population. 
There are several limitations in this study. Data were mainly extracted from records due to reasons in confidentiality resulting in a significant amount of incomplete and missing data and inaccuracies in anthropometric values. Ours was a cross sectional study; a prospective study would have been the best study design to assess the effect of HIV and HAART therapy on metabolic parameters. HIV associated lipodystrophy syndrome, known to be present sometimes in up to $80 \%$ of the patients on HAART therapy $(16,19)$, and having higher correlation with insulin resistance and metabolic abnormalities was not assessed in our study. Its presence or absence would have had a greater influence on some of our metabolic parameters.

In conclusion this study highlights the high prevalence of impaired fasting glucose in patients followed up at the National STD and AIDS campaign and the strong association of HARRT therapy with dyslipidaemia in the same group. It also emphasizes the necessity of proper treatment and follow-up of HIV positive patients with diabetes. This data would probably help in planning out management strategies for the above group of patients and also would help in carrying out similar studies on HIV patients on a national level.

\section{References}

1. Larsson R, Eckert -Norton M, Colagreco JP, et al. Disorders of glucose metabolism in the context of human immunodeficiency virus infection. JAANP 2006; 18: 92-103.

2. Dagogo J. New drugs and diabetes risk: antipsychotic and antiretroviral agents. In clinical diabetes. Fonseca VA, ED. Philadelphia, Saunders, 2006: 569-81.

3. Brown TT, Kingsley LA, Palella FJ. et al. Antiretroviral therapy and the prevalence and incidence of diabetes in multicenter AIDS cohort study. Arch Intern Med 2005; 165: 1179-84.

4. Waskin H, Helmick CG, Sattle FR. Risk factors for hyperglycaemia associated with Pentamidine therapy for Pneumocystis pneumonia. JAMA 1988; 260: 345-7.

5. Henry K, Sullivan C, McCabe K. Diabetes mellitus induced by megesterol acetate in patients with AIDs and cachexia. Ann Intern Med 1992; 116: 53-4.

6. Leonardo Calza L, Roberto Manfredi R, Chiodo F. Dyslipidaemia associated with antiretroviral therapy in HIVinfected patients. Journal of Antimicrobial Chemotherapy 2004; 53: 10-14.

7. Grunfeld C. Dyslipidaemia and its Treatment in HIV Infection. Top HIV Med 2010; 18(3): 112-18.

8. National STD/AIDS control programme, Sri Lanka (2013). Reported and estimated HIV cases. Retrieved from www.aidscontrol.gov.lk.
9. Standards of Medical Care in Diabetes. 2012 Diabetes Care, Volume 35, Supplement 1, January 2012.

10. Third Report of the National Cholesterol Education Program (NCEP) Expert Panel on Detection, Evaluation, and Treatment of High Blood Cholesterol in Adults (Adult Treatment Panel III) National Cholesterol Education Program. National Heart, Lung, and Blood Institute National Institutes of Health NIH Publication No. 02-5215 September 2002.

11. WHO/IASO/IOTF. The Asia Pacific Perspective: Redefining Obesity and Its Treatment. Health Communications Australia Pty Ltd, 2000.

12. Katulanda P, Constantine GR, Mahesh RG. Prevalence and Projections of Diabetes and Prediabetes in Sri Lanka. Sri Lanka Diabetes, Cardiovascular Study. Diab Med 2008; 25: 1062-9.

13. Wit S, Sabin C, Weber. Incidence and Risk Factors for NewOnset Diabetes in HIV-Infected Patients. The Data Collection on Adverse Events of Anti-HIV Drugs (D. A. D) Study. Diabetes Care 2008; 31: 1224-9.

14. Calza L, Masetti G, Piergentili B. Prevalence of diabetes mellitus, hyperinsulinaemia and metabolic syndrome among 755 adult patients with HIV-1 infection. Intl J STD AIDS 2011; 22(1): 43-5.

15. Iseuzo SA, Makusidi MA. Metabolic dysfunctions in nonantiretroviral treated HIV/AIDS patients. Niger J Clin Practice 2009; 12(4): 375-8.

16. Hadigan C, Meigs JB, Corcoran C, et al. Metabolic abnormalities and cardiovascular disease risk factors in adults with human immunodeficiency virus infection and lipodystrophy. Clin Infect Dis 2001; 32: 130-9.

17. Jones CY, Wilson IB, Greenberg AS, et al. Insulin resistance in HIV-infected men and women in the nutrition for healthy living cohort. J. Acquir. Immune Defic. Syndr 40: 202-11.

18. De Silva F, Bassichetto K, Lewi D. Lipid Profile, Cardiovascular Risk Factors and Metabolic Syndrome in a Group of AIDS Patients. Arq Bras Cardiol 2009; 93(2): 107-111.

19. Klotsas E, Klotsas A. HIV and HIV Treatment: Effects on Fats, Glucose and Lipids. British Medical Bulletin 2007; 84: 49-68.

20. Tien P, Schneider M, Cole S, et al. Antiretroviral Therapy Exposure and Insulin Resistance in the Women's Interagency HIV Study. J Acquir Immune Defic Syndr 2008; 49(4): 369-76.

21. Brown TT, Li X, Cole SR, et al. Cumulative exposure to nucleoside analogue reverse transcriptase inhibitors is associated with insulin resistance markers in the Multicenter AIDS Cohort Study. AIDS 2005; 19: 1375-83.

22. Da Silva E, Bassichetto K, Lewi D, et al. Lipid profile, cardiovascular risk factors and metabolic syndrome in a group of AIDS patients. Arq Bras Cardiol 2009; 93(2): 107-111. 
23. Homasanit M, Nelson KE, Sonjai A. Body shape and metabolic abnormalities in Thai HIV-infected patients. AIDS Res Hum Retroviruses 2007; 23(11): 1314-21.

24. Sprinz E, Lazzaretti RK, Kuhmmer R. Dyslipidaemia in HIV-infected individuals. Braz J Infect Dis 2010; 14(6): 575-88.

25. Katulanda P, Jayawardena MA, Sheriff MH. Prevalence of overweight and obesity in Sri Lankan adults. Obes Rev 11(11): 751-6.

26. Worm S, Sabin C, Reiss P, et al. Presence of the metabolic syndrome is not a better predictor of cardiovascular disease than the sum of its components in HIV-infected individuals data collection on adverse events of Anti-HIV drugs
(D.A.D) study. Diabetes Care 2009; 32: 472-90.

27. Malaza A, Mossong J, Ba“rnighausen T, Newell ML. (2012) Hypertension and obesity in adults living in a high HIV prevalence rural area in South Africa. PLoS ONE 7(10): e47761. doi:10.1371/journal.pone.0047761

28. Kroll AF, Sprinz E, Leal SC, Labrêa Mda G, Setúbal S. Prevalence of Cardiovascular Risk and Obesity Among HIV/ AIDS in Porto Alegre Brazil. Arq Bras Endocrinol Metabol 2012; 56(2): 137-41.

29. Samaras K. Metabolic consequences and therapeutic options in highly active antiretroviral therapy in human immunodeficiency virus-1 infection. Journal of Antimicrobial Chemotherapy 2008; 61: 238-45. 